\title{
Nonlinear optical signatures of the tensor order in $\mathrm{Cd}_{2} \mathrm{Re}_{2} \mathrm{O}_{7}$
}

\author{
JESSE C. PETERSEN ${ }^{1}$, MICHAEL D. CASWELL ${ }^{1}$, J. STEVEN DODGE ${ }^{1 *}$, IVAN A. SERGIENKO ${ }^{2,3}$, JIAN HE ${ }^{2,3 \dagger}$, \\ RONGYING JIN²,3 AND DAVID MANDRUS 2,3 \\ ${ }^{1}$ Department of Physics, Simon Fraser University, Burnaby, British Columbia V5A 1S6, Canada \\ ${ }^{2}$ Materials Science and Technology Division, Oak Ridge National Laboratory, Oak Ridge, Tennessee 37831, USA \\ ${ }^{3}$ Department of Physics and Astronomy, The University of Tennessee, Knoxville, Tennessee 37996, USA \\ ${ }^{\dagger}$ Present address: Department of Physics and Astronomy, Clemson University, Clemson, South Carolina 29631, USA \\ *e-mail: jsdodge@sfu.ca
}

6 rowing evidence indicates that the superconducting pyrochlore $\mathrm{Cd}_{2} \mathrm{Re}_{2} \mathrm{O}_{7}$ exhibits a structural phase transition at $T_{\mathrm{c}}=200 \mathrm{~K}$ with an unusual tensor character ${ }^{1-3}$. The structural order parameter for this state is two-dimensional, and spanned by distinct but nearly degenerate crystallographic structures $I 4_{1} 22$ and $I \overline{4} m 2$ (ref. 1 ). Symmetry rules imply that the low-energy excitations of the ordered state are Goldstone phonons, or long wavelength fluctuations between the two crystal structures. These are the structural equivalents of magnons in an $\mathrm{XY}$ antiferromagnet, with the two crystal structures analogous to orthogonal spin directions in the $x y$-plane. Goldstone phonons have been observed in Raman spectroscopy ${ }^{3}$, but high-resolution X-ray and neutron scattering experiments have produced conflicting assignments of the static low-temperature structure ${ }^{4-6}$. Here, we use optical secondharmonic generation with polarization sensitivity to assign the $I \overline{4} m 2$ structure unambiguously and verify an auxiliary condition on the structure that is implied by the order parameter symmetry. We also show that the temperature dependence of the order parameter is consistent with thermal occupation of the Goldstone mode. The methodology may be applied more widely in characterizing ordered states in matter.

Various experimental probes show a continuous cubic-totetragonal transition in $\mathrm{Cd}_{2} \mathrm{Re}_{2} \mathrm{O}_{7}$ at $T_{\mathrm{c}}$ (refs 1,2,4-10). However, as Anderson and Blount pointed out over 40 years $\mathrm{ago}^{11}$, a less conventional order parameter, possibly with ferroelectric character, must accompany strain to make any cubic-to-tetragonal transition continuous. Experiments have indeed ruled out strain as the primary order parameter for $\mathrm{Cd}_{2} \mathrm{Re}_{2} \mathrm{O}_{7}$ (ref. 2), and both X-ray and neutron diffraction reveal broken inversion symmetry below $T_{\mathrm{c}}$ (refs 4-6). Theoretical analysis indicates that the true order parameter is a second-rank pseudotensor, corresponding to the $E_{u}$ representation of the cubic point group ${ }^{1,2}$, shown in Fig. 1. The more familiar types of order all have lower rank: vectors for ferroelectricity, inversion-symmetric pseudovectors for ferromagnetism and second-rank tensors for ferroelasticity. Like the vector order of ferroelectricity, $E_{u}$ tensor order in
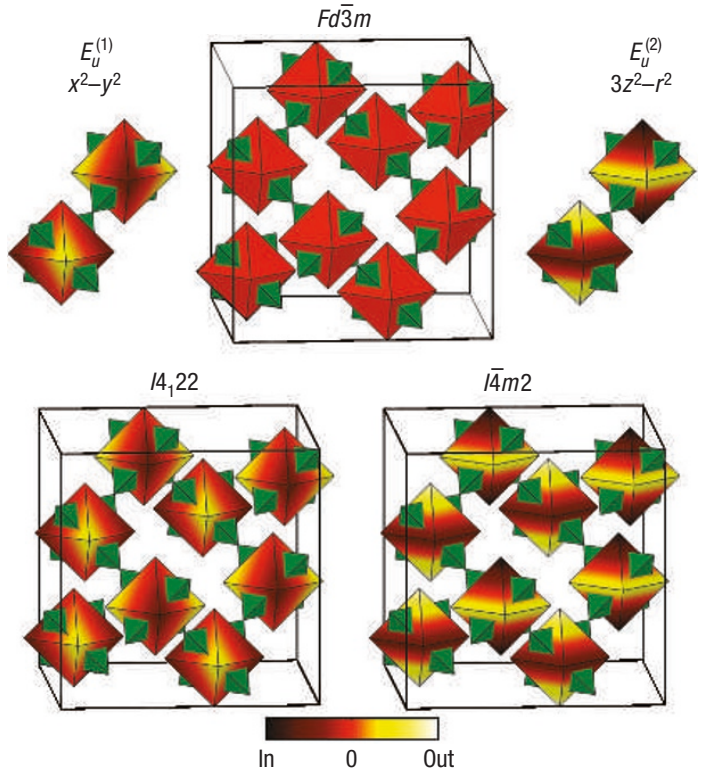

Figure 1 The structural phase transition of $\mathbf{C d}_{2} \mathbf{R e}_{2} \mathbf{0}_{7}$. (Top centre) Cubic pyrochlore unit cell. The vertices of green tetrahedra indicate Re sites and red octahedra indicate 01 sites. $\mathrm{Cd}$ and 02 sublattices are not shown. (Top left and right) Distortions of neighbouring 01 octahedra associated with the two-fold degenerate $E_{u}$ order parameter. Colour indicates oxygen displacement from the octahedron centre. Point group labels are shown together with the polynomial representation of a single octahedral distortion. Neighbouring octahedra have displacements with opposite sign, thus breaking inversion symmetry. (Bottom left and right) Pseudocubic unit cells of the distorted lattices, labelled by crystallographic group.

$\mathrm{Cd}_{2} \mathrm{Re}_{2} \mathrm{O}_{7}$ breaks inversion symmetry, but it is piezoelectric, not ferroelectric. 

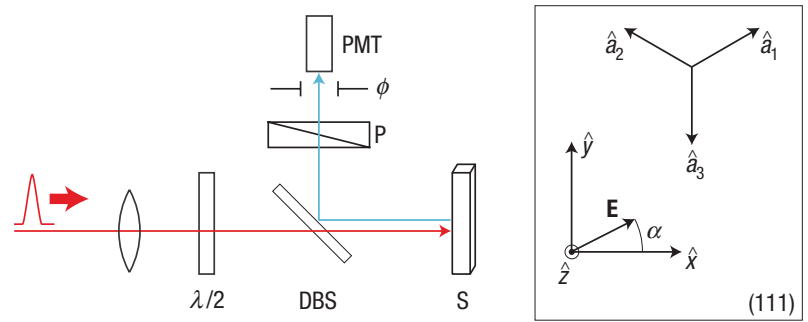

Figure 2 Experimental geometry. (Left) Schematic diagram of the measurement system used for second-harmonic ellipsometry. Femtosecond laser pulses with $\hbar \bar{\omega}=1.5 \mathrm{eV}$ were focused through a dichroic beamsplitter (DBS) to a $100 \mu \mathrm{m}$-diameter spot on a cubic (111) surface of the sample (S); the reflected SHG is detected by a photomultiplier tube (PMT) after passing through an iris $(\phi)$. Input and output polarization states are specified with a half-wave plate $(\lambda / 2)$ and a crystal polarizer (P), respectively. (Right) Cubic crystallographic system $\left(\hat{a}_{1}, \hat{a}_{2}, \hat{a}_{3}\right)$ of the high-temperature phase and optical coordinate system $(\hat{x}, \hat{y}, \hat{z})$, viewed along the optical axis.

Our analysis of current experiments limits the possible lowtemperature crystal structure to $I 4_{1} 22$ or $I \overline{4} m 2$, but no further; details are provided in the Methods section. These two structures must be nearly degenerate to yield a Goldstone mode, but an infinitesimal anisotropy will introduce a small gap in the excitation spectrum and select a well-defined static structure ${ }^{12}$. At energies well above this gap, the dispersion relation of the Goldstone boson is expected to be linear ${ }^{3}$, like the spin-wave spectrum of an antiferromagnet. At temperatures above the anisotropy scale, thermal occupation of Goldstone bosons is expected to degrade the static order quadratically with temperature, just as spin waves degrade the sublattice magnetization of an antiferromagnet ${ }^{13}$.

The tensor order of $\mathrm{Cd}_{2} \mathrm{Re}_{2} \mathrm{O}_{7}$ has been difficult to study experimentally because it is characterized by small atomic displacements, crystallographic twinning and high tensor rank. These challenges are well met by nonlinear optics. It is impossible to observe any d.c. piezoelectric effect in $\mathrm{Cd}_{2} \mathrm{Re}_{2} \mathrm{O}_{7}$ because it is a metal; nonetheless, the electronic transport properties are extremely sensitive to the structural transition, so we may expect similar sensitivity in states probed optically, where resonant enhancement from $\mathrm{O} \rightarrow \mathrm{Re}$ interband transitions are also expected to play a role $\mathrm{e}^{14,15}$. Nonlinear optical susceptibilities are characterized by tensors of third or higher rank, so they can reveal symmetries that are hidden from other techniques. Second-harmonic generation (SHG) is commonly used to probe broken inversion symmetry, both at interfaces and in bulk materials ${ }^{16,17}$. More recently it has been used to characterize magnetic symmetries ${ }^{18,19}$. Furthermore, its high spatial resolution facilitates measurements in the presence of domain structure ${ }^{20}$.

In the electric dipole approximation, the nonlinear optical susceptibility for SHG is a third-rank tensor $\chi_{j k l}$ defined by

$$
\mathcal{P}_{j}(2 \omega)=\chi_{j k l}(2 \omega ; \omega, \omega) E_{k}(\omega) E_{l}(\omega),
$$

where $\omega$ is the incident optical frequency, $\mathscr{P}(2 \omega)$ is the second-order nonlinear contribution to the polarization and $\mathbf{E}(\omega)$ is the incident electric field amplitude. If equation (1) is to remain invariant under inversion, $\chi_{j k l}$ must be inversionantisymmetric, reflecting broken inversion symmetry in the medium. In general $\chi_{j k l}$ has 27 complex independent elements, but experimental and crystal symmetries can reduce this number dramatically $^{16}$. Application of the appropriate permutation and point group symmetry operations to equation (1) then gives

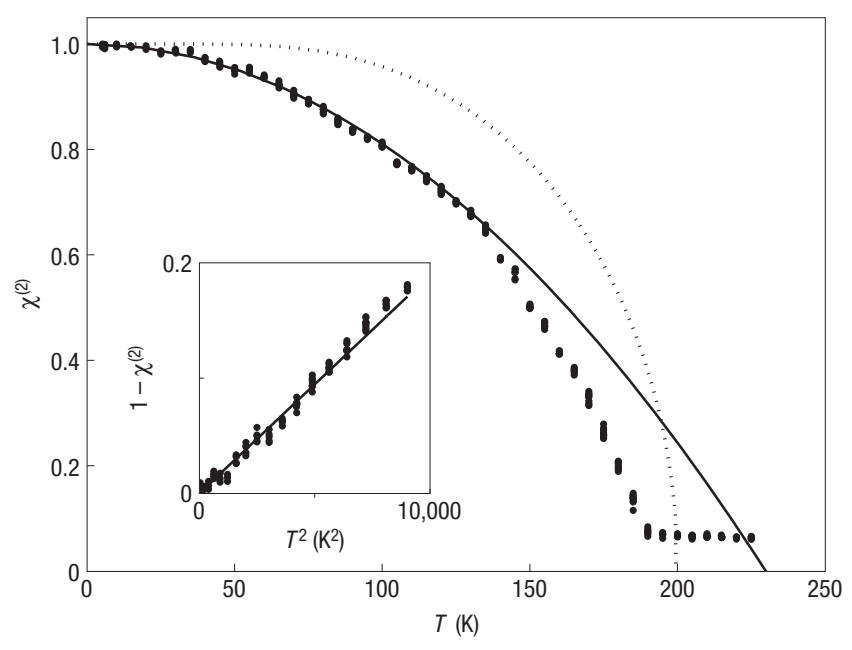

Figure 3 Temperature dependence of $\chi^{(2)}(T)$, measured by warming the sample from $T=5 \mathrm{~K}$ with $\alpha_{\omega}=0$ and with the polarizer removed. The mean-field prediction is shown as a dotted line, and the solid line shows $\chi^{(2)}(T)=1-\left(T / T_{\beta}\right)^{2}$, expected from thermal occupation of the Goldstone mode. We obtain $T_{\beta}=230 \pm 9 \mathrm{~K}$ by carrying out least-squares fits over a range $5 \mathrm{~K} \leq T \leq T_{\max }$, with $T_{\max }$ varying from $25-150 \mathrm{~K}$, then taking the mean and standard deviation of the parameter distribution. Repeated measurements at the same temperature indicate the statistical variation in the data. The inset shows the data and fit from the main panel, plotted as $1-\chi^{(2)}$ versus $T^{2}$.

$\chi_{123}=\chi_{132}=-\chi_{213}=-\chi_{231}$ as the only non-zero Cartesian tensor elements for $I 4_{1} 22$; similarly $\chi_{123}=\chi_{132}=\chi_{213}=\chi_{231}, \chi_{312}=\chi_{321}$ for $I \overline{4} m 2$. Thus, a single complex number determines the nonlinear susceptibility of crystals with $I 4_{1} 22$ symmetry, and in general two complex numbers are required for $I \overline{4} m 2$.

Figure 2 shows our experimental geometry. For concreteness we take the tetragonal $c$ axis parallel to the cubic $\hat{a}_{3}$ axis, and define the polar axis associated with the angle $\alpha$ to be orthogonal to it. In practice, the $c$ axis must be determined at low temperatures as described in the Methods section. In both crystallographic groups, the nonlinear polarization along one cubic direction is driven by the product of the electric fields along the other two. In the measurements discussed below, we find that when the SHG intensity is maximized, the input polarization $\alpha_{\omega}$ and the output analyser angle $\alpha_{2 \omega}$ are indeed perpendicular.

Figure 3 shows the temperature dependence of the normalized SHG amplitude, $\chi^{(2)}(T) \equiv \sqrt{P_{2 \omega}(T) / P_{2 \omega}(5 \mathrm{~K})}$, expected to be proportional to the order parameter amplitude. The clear onset below $T_{\mathrm{c}}$ directly implies that the order parameter breaks inversion symmetry. Polarization analysis indicates that the non-zero $\chi^{(2)}$ above $T_{c}$ results from the cubic sample surface and is unrelated to the $E_{u}$ order at lower temperatures. At low temperatures, the ordered state degrades much more rapidly than mean-field theory would predict $^{4}$, behaviour that we associate with the thermal occupation of Goldstone bosons.

A detailed theory for these novel lattice excitations does not yet exist. However, we can appeal to the analogy with antiferromagnetic spin waves to predict that at low temperatures $\chi^{(2)}=1-\left(T / T_{\beta}\right)^{2}$ with $T_{\beta}$ comparable to the theoretical meanfield $T_{\mathrm{c}}$ (ref. 13). Figure 3 shows that the agreement of this model with our data is superb. Our estimate of $T_{\beta}=230 \pm 9 \mathrm{~K}$ is somewhat above the actual $T_{c}$, as expected for the theoretical mean-field value. We expect this temperature dependence to only be valid at low temperatures, for which occupation densities are low and nonlinear mode coupling effects are minimized. That the data follow this 

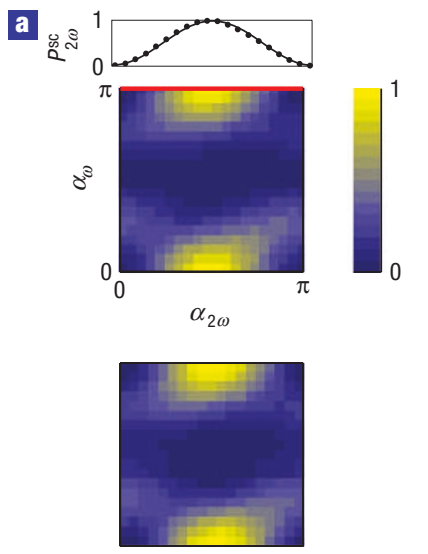

b
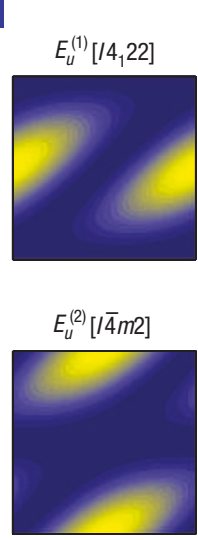

$\mathbf{G}$
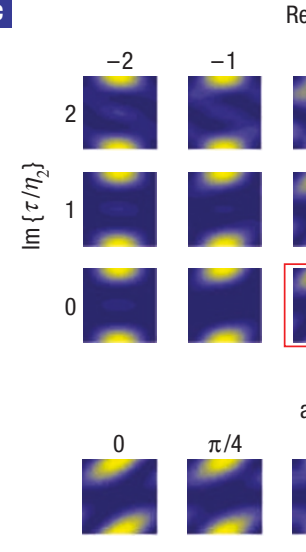

$\operatorname{Re}\left\{\tau / \eta_{2}\right\}$
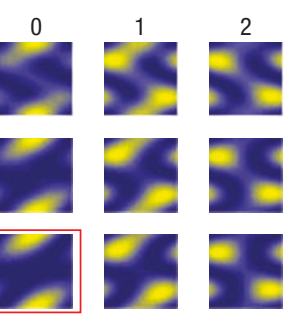

$\arg (\Phi)$
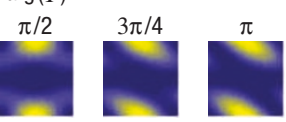
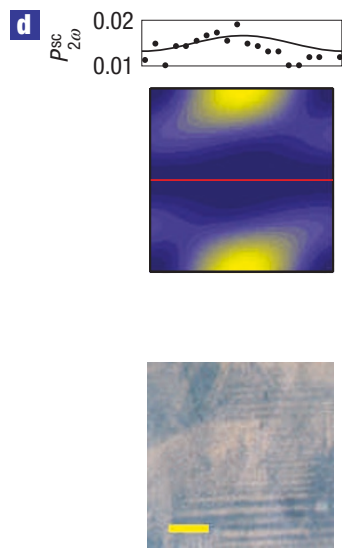

Figure 4 Second-harmonic ellipsometry plots. a, Scaled SHG power $P_{2 \omega}^{\text {sc }}\left(\alpha_{\omega}, \alpha_{2 \omega}\right)$ obtained at $T=135 \mathrm{~K}$ (top) and at $T=4 \mathrm{~K}$ (bottom). Also shown are data points and a model curve $\left(\eta_{1}=\tau=0, \Phi=1\right)$ along the line cut indicated in red, $P_{2 \omega}^{\text {sc }}\left(\alpha_{\omega}=\pi, \alpha_{2 \omega}\right)$ at $T=135 \mathrm{~K}$. b. Model calculations $(\tau=0, \Phi=1)$ for the two $E_{u}$ basis states. c. Model calculations for the $\overline{4} 2 m$ point group with various values of $\tau$ and $\Phi$. Top: $\Phi=1, \tau / \eta_{2}$ varying over the complex plane, with $\tau / \eta_{2}=0$ indicated with a red square. Substituting $\tau / \eta_{2} \rightarrow\left(\tau / \eta_{2}\right)^{*}$ yields identical results. Bottom: $\tau / \eta_{2}=0,|\Phi|=1$ and $\arg (\Phi) \in[0, \pi]$. Variation with $|\Phi|$ is much weaker. d, Top: Best-fit model of the $2 \mathrm{D}$ data set from $T=135 \mathrm{~K}$, shown with experimental data and the model curve from a cut along the red line $P_{2 \omega}^{\text {sc }}\left(\alpha_{\omega}=\pi / 2, \alpha_{2 \omega}\right)$. Bottom: Polarized white light micrograph of the tetragonal domain structure at $T=77 \mathrm{~K}$. The scale bar represents $100 \mu \mathrm{m}$.

temperature dependence up to nearly three-quarters of $T_{\mathrm{c}}$ indicates that the Goldstone bosons interact weakly, and that unlike spin waves these lattice modes may be treated quasiclassically.

The SHG polarization analysis shown in Fig. 4 now enables us to both assign the low-temperature crystallographic symmetry and associate it clearly with the $E_{u}$ order parameter. As noted above, the nonlinear susceptibility is determined by one or two complex parameters, depending on the crystal symmetry. To measure these, a typical experimental approach is to construct a high-symmetry geometry that yields one or two real parameters from a fit to a one-dimensional (1D) data set such as the line cuts shown in Fig. 4. However, additional polarization effects can result from linear birefringence, crystal twinning and small experimental misalignments. These will all tend to corrupt an analysis that assumes a more constrained experimental geometry. Also, distinct physical symmetries often are not naturally expressed in the experimental coordinates, so multiple effects can contribute to the signal variation when a single experimental parameter changes. We have developed an approach that uses 2D data sets obtained by varying $\alpha_{\omega}$ and $\alpha_{2 \omega}$, together with an analysis based on the irreducible group representations of the physical symmetries ${ }^{17,21}$.

In the usual notation for the representations of the cubic point group $^{22}$, the susceptibility in the $I 4_{1} 22$ crystal structure transforms as $E_{u}^{(1)}$, whereas in $I \overline{4} m 2$ the susceptibility transforms as the direct sum $E_{u}^{(2)} \oplus A_{2 u}$. We can express this decomposition in Cartesian form by associating the complex parameters $\eta_{1}, \eta_{2}$ and $\tau$ with $E_{u}^{(1)}, E_{u}^{(2)}$ and $A_{2 u}$ respectively. In the experimental geometry of Fig. 2, this gives a nonlinear polarization

$$
\mathfrak{P}_{\perp} \propto \frac{\eta_{1}}{\sqrt{3}}\left(\begin{array}{c}
-E_{y}^{2} \\
E_{x} E_{y}
\end{array}\right)+\eta_{2}\left(\begin{array}{c}
-E_{x} E_{y} \\
E_{x}^{2}
\end{array}\right)-\frac{\tau}{2}\left(\begin{array}{c}
2 E_{x} E_{y} \\
E_{x}^{2}-E_{y}^{2}
\end{array}\right),
$$

where $\mathscr{P}_{\perp}$ indicates that we have projected out the non-radiating component that lies along the optical axis. Neglecting optical anisotropy, the radiated SHG field will be proportional to $\mathscr{P}_{\perp}$. For $E_{u}$ order, linear birefringence is expected to appear as a secondary order parameter with $E_{g}^{(2)}$ symmetry, just as strain does. We model this birefringence by introducing a phenomenological complex parameter $\Phi$, and letting the radiated SHG field be $\mathbf{E}_{2 \omega} \propto\left(\mathcal{P}_{x}, \Phi \mathcal{P}_{y}\right)$. We find similar freedom in $\mathbf{E}_{\omega}$ unnecessary.
For $\alpha_{\omega}=\{0, \pi\}$, the incident polarization is along $\hat{x}$, so $E_{y}=0$ and we expect no SHG at all from the $I 4_{1} 22$ crystal structure, even in the presence of birefringence. Instead, we see a sinusoidal variation of the SHG power that has a maximum at $\alpha_{2 \omega}=\pi / 2$, perpendicular to the incident polarization. We can conclude immediately from this observation that order parameter anisotropy favours the low-temperature crystallographic symmetry $I \overline{4} m 2$. However, theoretical plots with $\tau=0$ and $\Phi=1$, shown in Fig. 4b, reveal subtle differences from the data that a complete description must accommodate. Figure $4 \mathrm{c}$ shows that the model variation with $\tau$ and $\arg (\Phi)$ is readily distinguished, and comparison with Fig. 4a shows that $\arg (\Phi)$ is more significant for characterizing the experimental data.

With the $E_{u}$ order parameter we expect $\tau \propto \eta_{2}^{3}$ to be small near $T_{c}$, even although it is allowed in the general $I \overline{4} m 2$ crystal symmetry ${ }^{1}$. This allows a further experimental test of the theory. We can associate $\tau / \eta_{2}$ directly with the SHG power variation over the line cut with $E_{x}=0$ in Fig. $4 \mathrm{~d}$. Consistent with $E_{u}$ order, a nonlinear least-squares fit to the $2 \mathrm{D}$ data set at $T=135 \mathrm{~K}$ yields best-fit parameters $\tau / \eta_{2} \cong 0.1+0.0 i$ and $\Phi \cong 1.1 \mathrm{e}^{1.3 i}$, together with a small constant offset $P_{2 \omega}^{0} \cong 0.013$ that we associate with surface SHG and instrumental background. The sensitivity to optical alignment creates systematic error that dominates statistical uncertainty, but contours of the $\chi^{2}$ surface are nearly circular in the complex $\tau / \eta_{2}$ plane and we can set a bound of 0.5 on $\left|\tau / \eta_{2}\right|$ through visual inspection of model plots such as those in Fig. 4. The model results shown in Fig. $4 \mathrm{~d}$ are in excellent agreement with experiment.

The properties of tensor order in $\mathrm{Cd}_{2} \mathrm{Re}_{2} \mathrm{O}_{7}$ exemplify the notion, pioneered by Landau and now firmly embedded in physics, that the low-energy properties of complex systems can be described with startling success by using just general symmetry principles and a few experimental inputs ${ }^{23}$. They may also serve as a foothold in reaching a better understanding of metals. One of the challenges for understanding how electrons travel in oxides is that the complexity of their interactions requires more sophisticated use of the standard principles of condensed-matter physics, and may even require the invention of new ones. With the structural aspects of the ordered state of $\mathrm{Cd}_{2} \mathrm{Re}_{2} \mathrm{O}_{7}$ now clearly identified and a guiding analogy with magnetism established, significant simplification has been 
achieved, and analysis of its intriguing electronic properties may proceed more effectively.

\section{METHODS}

\section{ANALYSIS OF CURRENT EXPERIMENTS}

Below $T_{\mathrm{c}}=200 \mathrm{~K} \mathrm{Cd}_{2} \mathrm{Re}_{2} \mathrm{O}_{7}$ loses three-fold rotation and inversion symmetries in a continuous ferrodistortive phase transition from an ideal pyrochlore structure, as discussed in ref. 2; the loss of inversion symmetry is further supported by the present work. Magnetic order on the rhenium sites is excluded by the absence of nuclear quadrupole resonance hyperfine splitting ${ }^{9}$. Sergienko and Curnoe ${ }^{1}$ used Landau theory to deduce that a continuous phase transition from $F d \overline{3} m$ to a non-magnetic state without inversion symmetry can only yield 14 possible space groups (their Table II). Of these, only $I 4_{1} 22$ and $I \overline{4} m 2$ are consistent with the Raman observation of a Goldstone mode ${ }^{3}$, as they are the only basis vectors of a single $O(n), n>1$ order parameter that exhibits no fourth-order anisotropy in the Landau free energy ${ }^{1}$.

Assignment of the low-temperature crystal structure from diffraction studies is ambiguous, and limited by the unusually small lattice displacements in the ordered state ${ }^{4-6}$. In the low-temperature phase, new X-ray diffraction peaks appear at $(0,0,10),(0,6,4)$ and at permutations of these cubic indices ${ }^{5}$. High-resolution X-ray measurements show further that the $(0,0,12)$ peak, allowed in the ideal pyrochlore structure, exhibits a weak splitting, whereas no splitting is resolved for the $(0,0,10)$ peak. In an untwinned crystal the $(0,0,10)$ tetragonal reflection is allowed in $I \overline{4} \mathrm{~m} 2$ and forbidden in $I 4_{1} 22$; this seems to be the basis for the $I \overline{4} m 2$ assignment in ref. 5 . The crystal is clearly twinned in this study, however, and in this case all of the peaks observed in ref. 5 are consistent with both $I 4_{1} 22$ and $I \overline{4} m 2$ (ref. 24). Subsequent powder neutron diffraction measurements were shown to be more consistent with $I \overline{4} m 2$ than with $F d \overline{3} m$ at low temperatures, but no comparison was made to $I 4_{1} 22$ (ref. 6 ). The role of twinning was recognized in ref. 4 , and an assignment to $I 4_{1} 22$ or $I 4_{1}$ was based on the recognition that the cubic $(0,0,10)$ peak would not split for these structures because the $(0,0,10)$ tetragonal reflection is forbidden. These measurements were resolution-limited, however, and there is nothing to prevent an approximate, non-space-group absence in the $I \overline{4} m 2$ structure. Given the unambiguous evidence for an $I \overline{4} m 2$ assignment provided here, it seems likely that this occurs.

In some experiments, an additional first-order transition is observed near $120 \mathrm{~K}$ (refs 25-30). There is evidence that this transition is induced by sample disorder ${ }^{29}$, and it is not consistently observed in crystals grown at Oak Ridge. We see no evidence of this transition in our experiments, as can be seen by comparing measurements at $T=5 \mathrm{~K}$ and $T=135 \mathrm{~K}$ in Fig. 4a. Nevertheless, if we assume that this transition is intrinsic, our results clearly exclude the currently favoured $I 4_{1} 22$ space group, but could be consistent with an F222 space group if the transition is primarily associated with the cadmium sublattice ${ }^{30}$.

\section{OPTICAL SECOND-HARMONIC GENERATION}

An optical second harmonic at $400 \mathrm{~nm}$ was detected with a blue-sensitive photomultiplier tube after passing the reflected light through dichroic filters. The properties of $\chi_{j k l}$ in $\mathrm{Cd}_{2} \mathrm{Re}_{2} \mathrm{O}_{7}$ dictate that we use a (111) cubic surface for study. At normal incidence to a cubic (100) surface, the nonlinear polarization would be along the optical axis and not radiate. We have verified that at the intensities used in this experiment, the SHG signal scales with the square of the incident power, as expected for a second-order optical nonlinearity. We have also confirmed with magnetic susceptibility measurements that $T_{\mathrm{c}}=200 \mathrm{~K}$ in this sample, about ten degrees above the apparent $T_{\mathrm{c}}$ in our measurements. This difference is independent of laser power, so it is not a simple heating effect, and the apparent $T_{\mathrm{c}}$ exhibits a hysteretic shift of about $5 \mathrm{~K}$ under temperature cycling that is not observed with other experimental probes. We tentatively attribute this behaviour to laser-induced domain formation.

\section{OPTICALALIGNMENT}

Optical alignment made use of the 2D data visualization shown in Fig. 4. By symmetry, the SHG power $P_{2 \omega}$ should satisfy $P_{2 \omega}\left(\pi-\alpha_{\omega}, \pi-\alpha_{2 \omega}\right)=$ $P_{2 \omega}\left(\alpha_{\omega}, \alpha_{2 \omega}\right)$. Deviations from this symmetry can be readily identified in the 2D data, indicating misalignment, and in practice this provides a stringent check that the angle of incidence is zero. We also identified the $\hat{c}$ axis with one of the three cubic axes by demanding that this symmetry be satisfied, after using X-rays to orient the $\mathrm{Cd}_{2} \mathrm{Re}_{2} \mathrm{O}_{7}$ crystal at room temperature. Finally, we have found that in highly twinned regions, diffracted SHG beams cause the structure of the $2 \mathrm{D}$ plot to change as a function of the detector iris diameter. This allowed us to confirm that we were observing a single tetragonal domain, after initial alignment based on correlating the laser spot position with polarized light microscopy images. This spot was located in a relatively large domain near the centre of the image shown in Fig. $4 \mathrm{~d}$ when the results in Fig. 4a were obtained. Although the incident spot diameter was $100 \mu \mathrm{m}$, optical nonlinearity reduces the region probed by SHG to $50 \mu \mathrm{m}$.

Received 11 April 2006; accepted 31 July 2006; published 27 August 2006.

References

1. Sergienko, I. A. \& Curnoe, S. H. Structural order parameter in the pyrochlore superconductor $\mathrm{Cd}_{2} \mathrm{Re}_{2} \mathrm{O}_{7}$. J. Phys. Soc. Jpn 72, 1607-1610 (2003).

2. Sergienko, I. A. et al. Metallic "ferroelectricity" in the pyrochlore $\mathrm{Cd}_{2} \mathrm{Re}_{2} \mathrm{O}_{7}$. Phys. Rev. Lett. 92, 065501 (2004).

3. Kendziora, C. A. et al. Goldstone-mode phonon dynamics in the pyrochlore $\mathrm{Cd}_{2} \mathrm{Re}_{2} \mathrm{O}_{7}$. Phys. Rev. Lett. 95, 125503 (2005).

4. Castellan, J. P. et al. Structural ordering and symmetry breaking in $\mathrm{Cd}_{2} \mathrm{Re}_{2} \mathrm{O}_{7}$. Phys. Rev. B 66, 134528 (2002).

5. Yamaura, J. \& Hiroi, Z. Low temperature symmetry of pyrochlore oxide $\mathrm{Cd}_{2} \mathrm{Re}_{2} \mathrm{O}_{7}$. J. Phys. Soc. Jpn $71,2598-2600$ (2002).

6. Weller, M. T., Hughes, R. W., Rooke, J., Knee, C. S. \& Reading, J. The pyrochlore family—a potential panacea for the frustrated perovskite chemist. Dalton Trans. 3032-3041 (2004).

7. Hanawa, M. et al. Superconductivity at $1 \mathrm{~K}$ in $\mathrm{Cd}_{2} \mathrm{Re}_{2} \mathrm{O}_{7}$. Phys. Rev. Lett. 87, 187001 (2001).

8. Jin, R. et al. Fluctuation effects on the physical properties of $\mathrm{Cd}_{2} \mathrm{Re}_{2} \mathrm{O}_{7}$ near $200 \mathrm{~K}$. J. Phys. Condens. Matter 14, L117-L123 (2002).

9. Vyaselev, O. et al. Superconductivity and magnetic fluctuations in $\mathrm{Cd}_{2} \mathrm{Re}_{2} \mathrm{O}_{7}$ via Cd nuclear magnetic resonance and Re nuclear quadrupole resonance. Phys. Rev. Lett. 89, 017001 (2002).

10. Sakai, H. et al. Low-temperature structural change and magnetic anomaly in superconducting $\mathrm{Cd}_{2} \mathrm{Re}_{2} \mathrm{O}_{7}$. Phys. Rev. B 66, 100509(R) (2002).

11. Anderson, P. W. \& Blount, E. I. Symmetry considerations on martensitic transformations: "Ferroelectric" metals? Phys. Rev. Lett. 14, 217-219 (1965).

12. Anderson, P. W. An approximate quantum theory of the antiferromagnetic ground state. Phys. Rev. 86, 694-701 (1952).

13. Kubo, R. The spin-wave theory of antiferromagnetics. Phys. Rev. 87, 568-580 (1952).

14. Singh, D. J., Blaha, P., Schwarz, K. \& Sofo, J. O. Electronic structure of the pyrochlore metals $\mathrm{Cd}_{2} \mathrm{Os}_{2} \mathrm{O}_{7}$ and $\mathrm{Cd}_{2} \mathrm{Re}_{2} \mathrm{O}_{7}$. Phys. Rev. B 65, 155109 (2002).

15. Harima, $\mathrm{H}$. Electronic bandstructures on $5 \mathrm{~d}$-transition metal pyrochlore: $\mathrm{Cd}_{2} \mathrm{Re}_{2} \mathrm{O}_{7}$ and $\mathrm{Cd}_{2} \mathrm{Os}_{2} \mathrm{O}_{7}$. J. Phys. Chem. Solids 63, 1035-1038 (2002).

16. Boyd, R. W. Nonlinear Optics 2nd edn (Academic, San Diego, 2003).

17. Plocinik, R. M., Everly, R. M., Moad, A. J. \& Simpson, G. J. Modular ellipsometric approach for mining structural information from nonlinear optical polarization analysis. Phys. Rev. B 72, 125409 (2005).

18. Hübner, W. H., Bennemann, K. H. \& Böhmer, K. Theory for the nonlinear optical response of transition metals: Polarization dependence as a fingerprint of the electronic structure at surfaces and interfaces. Phys. Rev. B 50, 17597-17605 (1994).

19. Fiebig, M., Pavlov, V. V. \& Pisarev, R. V. Second-harmonic generation as a tool for studying electronic and magnetic structures of crystals: Review. J. Opt. Soc. Am. B 22, 96-118 (2005)

20. Fiebig, M., Lottermoser, T., Fröhlich, D., Goltsev, A. V. \& Pisarev, R. V. Observation of coupled magnetic and electric domains. Nature 419, 818-820 (2002)

21. Jerphagnon, J., Chemla, D. \& Bonneville, R. The description of physical properties of condensed matter using irreducible tensors. Adv. Phys. 27, 609-650 (1978).

22. Tinkham, M. Group Theory and Quantum Mechanics (International Series in Pure and Applied Physics, McGraw-Hill, New York, 1964).

23. Anderson, P. W. Basic Notions of Condensed Matter Physics (Benjamin-Cummings, Menlo Park, 1984)

24. Hahn, T. (ed.) International Tables for Crystallography 5 th edn, Vol. A (Kluwer, Dordrecht, 2002).

25. Hiroi, Z., Yamaura, J., Muraoka, Y. \& Hanawa, M. Second phase transition in pyrochlore oxide $\mathrm{Cd}_{2} \mathrm{Re}_{2} \mathrm{O}_{7}$. J. Phys. Soc. Jpn 71, 1634-1636 (2002).

26. Hiroi, Z. \& Hanawa, M. Superconducting properties of the pyrochlore oxide $\mathrm{Cd}_{2} \mathrm{Re}_{2} \mathrm{O}_{7}$. J. Phys. Chem. Solids 63, 1021-1026 (2002).

27. Arai, K. et al. Structural transition in the pyrochlore superconductor $\mathrm{Cd}_{2} \mathrm{Re}_{2} \mathrm{O}_{7}$ observed by $\mathrm{Re}$ nuclear quadrupole resonance. J. Phys. Condens. Matter 14, L461-L466 (2002).

28. Barišić, N. et al. Electrical properties of $\mathrm{Cd}_{2} \mathrm{Re}_{2} \mathrm{O}_{7}$ under pressure. Phys. Rev. B 67, 245112 (2003).

29. Lu, C. et al. Imperfection-driven phase transition at $120 \mathrm{~K}_{\text {in }} \mathrm{Cd}_{2} \mathrm{Re}_{2} \mathrm{O}_{7}$. Phys. Rev. B 70, 092506 (2004).

30. Knee, C. S. et al. Order-disorder-order phase transitions in the pyrochlore superconductor $\mathrm{Cd}_{2} \mathrm{Re}_{2} \mathrm{O}_{7}$. Phys. Rev. B 71, 214518 (2005)

\section{Acknowledgements}

Research at SFU was supported by the National Science and Engineering Research Council of Canada, the Canadian Institute for Advanced Research Program in Quantum Materials, the Sloan Foundation and the Research Corporation. Research at Oak Ridge/UT is sponsored by the Division of Materials Sciences and Engineering, Office of Basic Energy Sciences, US Department of Energy, under contract DE-AC05-00OR22725 with Oak Ridge National Laboratory, managed and operated by UT-Battelle, LLC. We thank Z.-G. Ye for use of his polarized light microscope; we also thank him, D. Broun,

B. Gaulin, I. Herbut, C. Kendziora, G. Leach and D. Singh for helpful discussions.

Correspondence and requests for materials should be addressed to J.S.D.

\section{Author contributions}

J.C.P. and J.S.D. conceived and designed the experiments: J.C.P. and M.D.C carried them out, with crystals prepared and characterized by J.H., R.J. and D.M. J.C.P., M.D.C., J.S.D. and I.A.S. contributed to the measurement analysis. J.C.P. and J.S.D. wrote the paper.

\section{Competing financial interests}

The authors declare that they have no competing financial interests.

Reprints and permission information is available online at http://npg.nature.com/reprintsandpermissions/ 